\title{
SSREnricher: A computational approach for large-scale identification of polymorphic microsatellites based on comparative transcriptome analysis
} \author{
Zongjun Du Corresp. 1 \\ ${ }^{1}$ College of Animal Science and Technology, Sichuan Agricultural University, Chengdu, Sichuan, CHINA \\ Corresponding Author: Zongjun Du \\ Email address: 14364@sicau.edu.cn
}

Wei Luo ${ }^{\text {Equal first author, } 1}$, Qing Wu ${ }^{\text {Equal first author, }{ }^{1} \text {, Lan Yang }}{ }^{1}$, Pengyu Chen ${ }^{1}$, Siqi Yang ${ }^{1}$, Tianzhu Wang ${ }^{1}$, Yan Wang ${ }^{1}$,

Microsatellite (SSR) markers are the most popular markers for genetic analyses and molecular selective breeding in plants and animals. However, the currently available methods to develop SSRs are relatively time-consuming and expensive. One of the most factors is low frequency of polymorphic SSRs. In this study, we developed a software, SSREnricher, which composes of six core analysis procedures, including SSR mining, sequence clustering, sequence modification, enrichment containing polymorphic SSR sequences, false-positive removal and results output and multiple sequence alignment. After running of transcriptome sequences on this software, a mass of polymorphic SSRs can be identified. The validation experiments showed almost all markers ( $>90 \%)$ that were identified by the SSREnricher as putative polymorphic markers were indeed polymorphic. The frequency of polymorphic SSRs identified by SSREnricher was significantly higher $(P<0.05)$ than that of traditional and HTS approaches. The software package is publicly accessible on GitHub [https://github.com/byemaxx/SSREnricher]. 


\section{SSREnricher: A computational approach for Large-scale}

3 Identification of Polymorphic Microsatellites Based on

4 Comparative Transcriptome Analysis

5

6

7
Wei Luo ${ }^{\#}$, Qing Wü, Lan Yang, Pengyu Chen, Siqi Yang, Tianzhu Wang, Yan Wang, Zongjun $\mathrm{Du}^{*}$

College of Animal Science and Technology, Sichuan Agricultural University, Chengdu, 611130, China

\# These authors contributed equally to this work.

Corresponding Author:

Zongjun Du

Sichuan Agricultural University (Chengdu Campus), 211 Huimin Road, Wenjiang District, Chengdu 611130, PR China.

Email address: 14364@sicau.edu.cn 


\section{Abstract}

26 Microsatellite (SSR) markers are the most popular markers for genetic analyses and molecular

27

28

29

30

31

32

33

34

35

36

37

38

\section{9}

40

41

42

43

44

45

46

47

48

49

50

51

52

53

54

55

56

57

58

59

60

61

62

63

selective breeding in plants and animals. However, the currently available methods to develop SSRs are relatively time-consuming and expensive. One of the most factors is low frequency of polymorphic SSRs. In this study, we developed a software, SSREnricher, which composes of six core analysis procedures, including SSR mining, sequence clustering, sequence modification, enrichment containing polymorphic SSR sequences, false-positive removal and results output and multiple sequence alignment. After running of transcriptome sequences on this software, a mass of polymorphic SSRs can be identified. The validation experiments showed almost all markers (> 90\%) that were identified by the SSREnricher as putative polymorphic markers were indeed polymorphic. The frequency of polymorphic SSRs identified by SSREnricher was significantly higher $(P<0.05)$ than that of traditional and HTS approaches. The software package is publicly accessible on GitHub [https://github.com/byemaxx/SSREnricher].

\section{Introduction}

Microsatellites or simple sequence repeats (SSRs) are tandem repeats of one to six nucleotides present in all eukaryotic genomes. Due to the high mutation rates, co-dominant inheritance, high abundance, reproducibility of microsatellite-based assays, extensive genome coverage, chromosome specific location, amenability to automation and high throughput genotyping (Parida et al., 2009), SSRs have emerged as one of the most popular genetic markers in a wide range of applications in population genetics (Filippi et al., 2015; Touma et al., 2019), conservation biology (Han and An, 2009; Kalia et al., 2011; Longwu et al., 2012), markerassisted selection (MAS) (Parida et al., 2009; Che et al., 2014; Lv et al., 2014) and other studies (Moore et al., 1999; Palmer et al., 2013; Parida et al., 2009). In addition, there is increasing evidence that SSRs can serve a functional role in the regulation of gene expression by affecting transcription, translational activity, DNA structure, and other metabolic activities (Palmer et al., 2013).

Despite the broad applicability of SSRs in animal and plant genetics, their development as genetic markers remains challenging in many species, especially in non-model organisms. The main reason is that they need to be developed de novo in most species being examined for the first time (Kalia et al., 2011). Traditionally, microsatellite marker development requires the construction of a genomic library enriched for repeated motifs, isolation and sequencing of microsatellite-containing clones, primer design, optimization of PCR amplification for each primer pair, and a polymorphism assessment in a few unrelated individuals (Squirrell et al., 2003). PCR-based isolation of microsatellite arrays (PIMA) (Lin and Chang, 2013) and fast isolation by AFLP of sequences containing repeat sites (FIASCO) (Hakki and Akkaya, 2000) have been devised; however, most of these techniques are inefficient, time-consuming, multifarious, and expensive (Abdelkrim et al., 2009). A survey of service providers indicated that high expense (approximately 5,000-10,000 USD) will deliver approximately 10 
64 polymorphic loci from anywhere between one to more than three months by traditional cloning 65 approaches (Abdelkrim et al., 2009).

66 With the wide application of high-throughput sequencing (HTS) technologies, especially for

67 transcriptome sequencing, the development of SSR markers using HTS-based approaches has

68 become a feasible alternative for many species (Touma et al., 2019). This method has

69 dramatically reduced the time and cost requirement for large-scale development of SSR markers

70 compared to traditional methods (Wu et al., 2014). Nevertheless, the frequency of polymorphic

71 SSR markers developed by this method has also been very low thus far (Che et al., 2014; Lv et

72 al., 2014; Yang et al., 2014), as most of the loci cannot be effectively applied in genetic analyses

73 and marker-assisted selective breeding. To our best knowledge, there are no reports addressing

74 the low frequency of polymorphic SSR marker development.

75 In this study, we designed a pipeline and integrated it into a user-friendly software called

76 SSREnricher, which can be used to identify polymorphic microsatellites on a large-scale using

77 Trinity (Grabherr et al., 2011) assembled transcript files.

78

\section{Materials and Methods}

\section{Architectural structure}

SSREnricher was developed in Python. Polymorphic SSRs enrichment was performed by mining (Thiel et al., 2003) was integrated into the software to mine the SSRs in the transcripts, and CDHIT (Li and Godzik, 2006) was used to cluster the transcripts. The program composes of six core analysis procedures, including those outlined in Fig. 2.

1) SSR mining: MISA is integrated into SSREnricher and is used to mine SSR sequence information in transcripts, using the following settings: SSR motifs and the number of repeats shown respectively, monomer-10, dimer-6, trimer-5, tetramer-5, pentamer-5, and hexamer-5. The maximal number of interrupting basepairs in a compound microsatellite is set to 100 . obtain clustering and positive or negative strand information of sequences.

\section{3) Sequence modification: The negative-strand sequences are converted into reverse} complementary sequences.

4) Enrichment containing polymorphic SSR sequences: The repeat units possessing only one nucleotide are filtered out. Repeat units less than 50bp from the beginning or end of the sequence are also removed. results. The clusters that contain sequences with multiple SSRs loci with the same motif, while others miss some SSRs loci, are removed.

6) Results output and multiple sequence alignment: Multi-sequence alignment is performed on the obtained clusters to observe the position of the SSRs and generates the result files. 
102 The software is supported on macOS and Linux, and is available in two different versions; one

103 with a graphical user interface allowing for easy use, and one in command-line version, allowing

104 for a more flexible and efficient use through the server.

105

106

\section{Identification of polymorphic SSRs using SSREnricher}

108

To validate our software, we used it to develop SSR markers from rice (Oryza sativa L.) and

109 grass carp (Ctenopharyngodon idella), the transcriptomes of which are publically available. Analysis in these two species allowed for the validation of our pipeline in both a plant and

111 The transcriptomes of rice and grass carp were acquired from the sequence read archive (SRA)

112 database of the National Center for Biotechnology Information (NCBI). Three rice

113 (SRR1799209, SRR1974265, and SRR2048540) and two carp (SRR1618540, SRR1618542)

114 transcriptomes obtained from different ecotypes were acquired. The transcriptomes were also

115 assembled using Trinity.

116

117 Validation experiments

118 Ten individuals of grass carp and rice were used for SSR validation. For carp, genomic DNA

119 was isolated from tail fin using a salt-extraction method with slight modifications. For rice, 120 genomic DNA was isolated from leaves using the DNeasy Plant mini prep kit (Qiagen). DNA 121 concentration for each sample was determined using NanoDrop (Thermo Scientific). Primers

122 were designed using Primer premier 5 software (Lalitha, 2000). All the PCR amplifications were 123 carried out in a $10 \mu \mathrm{L}$ volume containing $1 \mu \mathrm{L} 10 \times$ buffer (with $\mathrm{Mg}^{2+}$ ), $100 \mu \mathrm{M}$ dNTPs, $0.5 \mu \mathrm{L}$ 124 primer pairs, $1 \mathrm{U}$ Taq DNA polymerase and $20 \mathrm{ng}$ genomic DNA. The reaction program was 5 $125 \mathrm{~min}$ at $95{ }^{\circ} \mathrm{C}$, followed by 30 cycles of $30 \mathrm{~s}$ at $94^{\circ} \mathrm{C}, 30 \mathrm{~s}$ at annealing temperature $57^{\circ} \mathrm{C}, 30 \mathrm{~s}$ at $12672{ }^{\circ} \mathrm{C}$ and a final extension at $72{ }^{\circ} \mathrm{C}$ for $8 \mathrm{~min}$; then stored at $4{ }^{\circ} \mathrm{C}$. The PCR products were 127 separated by size with Genescan-400 HD size ladders (Applied Biosystems; Foster City, USA), 128 by capillary gel electrophoresis using the ABI 3730 Genetic Analyser (Applied Biosystems). The 129 peak heights and fragment sizes were analyzed using GeneMarker software (Holland and Parson, 130 2011).

131

\section{Results}

133 Three rice and two carp transcriptomes were used to enrich for polymorphic SSRs. The total 134 number of SSRs identified by MISA (non-including mononucleotides) was 9,901 in rice and 13516,700 in carp (Table 1). After enrichment using our pipeline, a total of 480 and 680 putatively 136 polymorphic SSRs were obtained, respectively. The putative polymorphic SSRs enriched by this 137 program were showed in Appendix file 1. Thirty of rice and 20 loci of grass carp were randomly 138 selected for primer design to test the polymorphism of potential SSRs. After PCR amplification 139 and agarose electrophoresis, $27(90 \%)$ and $20(100 \%)$ of the primers could amplify the sequence 140 with distinct product bands in rice and grass carp, respectively. Of which, 26 (96.30\%) and 19 
$141(95.00 \%)$ loci showed to be polymorphic. The detail information of polymorphic SSRs validated

142 by experiments was showed in Appendix file 2.

143 We compared the HTS approach (Wang et al., 2011) and our pipeline for polymorphic SSRs

144 detection using different transcriptomes in grass carp and rice. The ratio of polymorphic SSRs

145 was raised more than three times. The highest frequency of polymorphic SSRs developed using

146 genomic sequences in rice was $73.6 \%$ (Zheng et al., 2008), while our pipeline provided a

147 frequency of up to $95 \%$.

148 We randomly recorded the polymorphic marker frequency in different species, as determined by

149 different methods, including the traditional approach and HTS. The frequency of polymorphic

150 SSRs identified by the traditional clone approach ranged from 5.92\% (Estuarine tapertail

151 anchovy (Coilia nasus)) to 30.00\% (Pseudogyrincheilus prochilus) with a mean of 12.04\%,

152 while the frequency of polymorphic SSRs divided from transcriptome sequenced by HTS ranged

153 from 9.20\% (Pigeonpea (Cajanus cajan)) to 73.60\% (Rice (Oryza sativa)) with a mean of

$15437.93 \%$. The validation experiment showed that the frequency of polymorphic SSRs identified

155 by SSREnricher was above $90 \%$ for both species, with a mean of $93.93 \%$. One-way ANOVA

156 indicated that the frequency of polymorphic SSRs identified by this method was significantly

157 higher than that identified by other methods $(P<0.05)$ (Fig. 3).

158

159 Discussion

160 As the previous reports, the frequency of polymorphic SSR marker development by traditional

161 methods and HTS approaches is relatively low (usually, the frequency ranges from 10\% to $70 \%$ ).

162 Thus, these approaches require a significant amount of time and money for primer design and

163 experimental validation by identifying and separating the many "monomorphic" SSRs from the

164 minority of the polymorphic ones (Kalia et al., 2011; Tang et al., 2008). In the present study, we

165 developed a software, SSREnricher, which can significantly increase the frequency of

166 polymorphic SSR markers. As demonstrated by the validation experiments in rice and grass carp,

167 the frequency of polymorphic SSR was increased to $>90 \%$.

168 SSREnricher was developed based on the idea of enriching homologous sequences containing

169 the same SSR with a different number of repeats and identifying polymorphic SSRs using

170 computational and bioinformatics analyses. Compared with traditional methods, SSREnricher

171 have eliminated the most intensive wet lab steps in a computational way, significantly reducing

172 the running time. In addition, the cost of our method remains much lower than that of

173 conventional methods. Similar to HTS, the most costly step of SSR marker development is the

174 de novo transcriptome sequencing when there is no available online sequence data in public

175 databases.

176 The factors that affect the frequency of polymorphic SSRs enriched by the SSREnricher are: 1)

177 genetic differentiation between samples. In theory, the larger genetic differentiation between

178 samples detected by this program, the more putative polymorphic SSRs will be enriched. Thus, it

179 is best not to use individuals with very close relationships, such as full- and half-sib families, to

180 enrich polymorphic SSRs using this program. 2) The specificity of the species itself. The 
181 frequency of identifying polymorphic SSRs depends on the species and the genotypes used for

182 the evaluation (Tang et al., 2008). According to a survey of SSR development using expressed

183 sequence tags (ESTs) in more than fifty species, the frequency of polymorphic SSRs varies

184 dramatically (Wu et al., 2014). As previously reported, microsatellite genesis is an evolutionarily

185 dynamic process and has proven to be exceedingly complex, and any change in SSRs resulting in

186 increase or decrease in repeat numbers is associated with mutation rate (Ellegren, 2004; Pearson

187 et al., 2005).

188 With the rapid development of sequencing technologies, the cost and time have been greatly

189 reduced, allowing for widespread use of high-throughput sequencing methods. The number of

190 sequenced and deposited transcriptomes in public databases for many economically important

191 species is continuously increasing. As a consequence, the large-scale development of SSR

192 markers at a low cost and within a short timescale is becoming a feasible task. Thus,

193 development of polymorphic SSR markers using SSREnricher has attractive potential

194 applications in the fields of genetic analyses and molecular selective breeding in plants and

195 animals.

196

197

198

199

\section{Conclusions}

Almost all tested SSRs predicted to be polymorphic by the SSREnricher are indeed polymorphic, indicating development of polymorphic SSR markers by the SSREnricher is efficient and

200 reliable. The frequency of polymorphic SSR markers can be significantly increased, and thus the

201 time and cost can be notably decreased by using the SSREnricher.

202

203

\section{References}

204

Abdelkrim, J., Robertson, B.C., Stanton, J.A.L., Gemmell, N.J., 2009. Fast, cost-effective

205

206

207

208

209 development of species-specific microsatellite markers by genomic sequencing. Biotechniques. https://doi.org/10.2144/000113084

Chabot, C.L., Nigenda, S., 2011. Characterization of 13 microsatellite loci for the tope shark, Galeorhinus galeus, discovered with next-generation sequencing and their utility for eastern Pacific smooth-hound sharks (Mustelus). Conserv. Genet. Resour. https://doi.org/10.1007/s12686-011-9402-y

Che, R., Sun, Y., Sun, D., Xu, T., 2014. Characterization of the miiuy croaker (Miichthys miiuy) transcriptome and development of immune-relevant genes and molecular markers. PLoS One. https://doi.org/10.1371/journal.pone.0094046

Csencsics, D., Brodbeck, S., Holderegger, R., 2010. Cost-effective, species-specific microsatellite development for the endangered dwarf bulrush (Typha minima) using nextgeneration sequencing technology. J. Hered. https://doi.org/10.1093/jhered/esq069

Dutta, S., Kumawat, G., Singh, B.P., Gupta, D.K., Singh, S., Dogra, V., Gaikwad, K., Sharma, T.R., Raje, R.S., Bandhopadhya, T.K., Datta, S., Singh, M.N., Bashasab, F., Kulwal, P., Wanjari, K.B., K Varshney, R., Cook, D.R., Singh, N.K., 2011. Development of genic-SSR 
220

221

222

223

224

225

226

227

228

229

230

231

232

233

234

235

236

237

238

239

240

241

242

243

244

245

246

247

248

249

250

251

252

253

254

255

256

257

258

259

markers by deep transcriptome sequencing in pigeonpea [Cajanus cajan (L.) Millspaugh]. BMC Plant Biol. https://doi.org/10.1186/1471-2229-11-17

Ellegren, H., 2004. Microsatellites: Simple sequences with complex evolution. Nat. Rev. Genet. https://doi.org/10.1038/nrg1348

Filippi, C. V., Aguirre, N., Rivas, J.G., Zubrzycki, J., Puebla, A., Cordes, D., Moreno, M. V., Fusari, C.M., Alvarez, D., Heinz, R.A., Hopp, H.E., Paniego, N.B., Lia, V. V., 2015. Population structure and genetic diversity characterization of a sunflower association mapping population using SSR and SNP markers. BMC Plant Biol. https://doi.org/10.1186/s12870-014-0360-x

Grabherr, M.G., Haas, B.J., Yassour, M., Levin, J.Z., Thompson, D.A., Amit, I., Adiconis, X., Fan, L., Raychowdhury, R., Zeng, Q., Chen, Z., Mauceli, E., Hacohen, N., Gnirke, A., Rhind, N., Di Palma, F., Birren, B.W., Nusbaum, C., Lindblad-Toh, K., Friedman, N., Regev, A., 2011. Full-length transcriptome assembly from RNA-Seq data without a reference genome. Nat. Biotechnol. https://doi.org/10.1038/nbt.1883

Hakki, E.E., Akkaya, M.S., 2000. Microsatellite isolation using amplified fragment length polymorphism markers: No cloning, no screening. Mol. Ecol. https://doi.org/10.1046/j.1365-294X.2000.11143.x

Han, J., An, L.Z., 2009. Isolation and characterization of microsatellite loci in Peganum harmala (Peganaceae), an important resist-drought and medicinal plant. Conserv. Genet. https://doi.org/10.1007/s10592-009-9849-5

Holland, M.M., Parson, W., 2011. GeneMarker® HID: A Reliable Software Tool for the Analysis of Forensic STR Data. J. Forensic Sci. https://doi.org/10.1111/j.15564029.2010.01565.x

Iorizzo, M., Senalik, D.A., Grzebelus, D., Bowman, M., Cavagnaro, P.F., Matvienko, M., Ashrafi, H., Van Deynze, A., Simon, P.W., 2011. De novo assembly and characterization of the carrot transcriptome reveals novel genes, new markers, and genetic diversity. BMC Genomics. https://doi.org/10.1186/1471-2164-12-389

Kalia, R.K., Rai, M.K., Kalia, S., Singh, R., Dhawan, A.K., 2011. Microsatellite markers: An overview of the recent progress in plants. Euphytica. https://doi.org/10.1007/s10681-0100286-9

Lalitha, S., 2000. Primer Premier 5. Biotech Softw. Internet Rep. https://doi.org/10.1089/152791600459894

Li, W., Godzik, A., 2006. Cd-hit: A fast program for clustering and comparing large sets of protein or nucleotide sequences. Bioinformatics. https://doi.org/10.1093/bioinformatics/bt1158

Lin, H.S., Chang, S. Bin, 2013. PCR-based isolation of microsatellite arrays (PIMA). Methods Mol. Biol. https://doi.org/10.1007/978-1-62703-389-3-3

Longwu, G., Cuiyun, L., Guangxiang, T., Chao, L., Wei, X., 2012. Development and characterization of twenty microsatellite markers for the endangered fish Luciobarbus capito. Conserv. Genet. Resour. https://doi.org/10.1007/s12686-012-9660-3 
260

261

262

263

264

265

266

267

268

269

270

271

272

273

274

275

276

277

278

279

280

281

282

283

284

285

286

287

288

289

290

291

292

293

294

295

296

297

298

Lv, J., Liu, P., Gao, B., Wang, Y., Wang, Z., Chen, P., Li, J., 2014. Transcriptome analysis of the Portunus trituberculatus: De novo assembly, growth-related gene identification and marker discovery. PLoS One. https://doi.org/10.1371/journal.pone.0094055

Moore, S.S., Whan, V., Davis, G.P., Byrne, K., Hetzel, D.J.S., Preston, N., 1999. The development and application of genetic markers for the Kuruma prawn Penaeus japonicus, in: Aquaculture. https://doi.org/10.1016/S0044-8486(98)00461-X

Palmer, M.E., Lipsitch, M., Moxon, E.R., Bayliss, C.D., 2013. Broad conditions favor the evolution of phase-variable loci. MBio. https://doi.org/10.1128/mBio.00430-12

Parida, S.K., Kalia, S.K., Kaul, S., Dalal, V., Hemaprabha, G., Selvi, A., Pandit, A., Singh, A., Gaikwad, K., Sharma, T.R., Srivastava, P.S., Singh, N.K., Mohapatra, T., 2009. Informative genomic microsatellite markers for efficient genotyping applications in sugarcane. Theor. Appl. Genet. https://doi.org/10.1007/s00122-008-0902-4

Pearson, C.E., Edamura, K.N., Cleary, J.D., 2005. Repeat instability: Mechanisms of dynamic mutations. Nat. Rev. Genet. https://doi.org/10.1038/nrg1689

Perry, J.C., Rowe, L., 2011. Rapid microsatellite development for water striders by nextgeneration sequencing. J. Hered. https://doi.org/10.1093/jhered/esq099

Santos, B.S., Romana-Eguia, M.R.R., Basiao, Z.U., Ikeda, M., 2015. Development and characterization of nine novel microsatellite markers for the milkfish Chanos chanos. Conserv. Genet. Resour. https://doi.org/10.1007/s12686-014-0393-3

Shi, F., Xu, N., Xiong, M., Wang, X., Yang, Z., Que, Y., Zhu, B., Hu, J., Chang, J., 2009. Isolation and characterization of 15 microsatellite loci in an endemic Chinese cyprinid fish, Pseudogyrincheilus prochilus, and their cross-species amplification in two related species. Conserv. Genet. Resour. https://doi.org/10.1007/s12686-009-9092-x

Squirrell, J., Hollingsworth, P.M., Woodhead, M., Russell, J., Lowe, A.J., Gibby, M., Powell, W., 2003. How much effort is required to isolate nuclear microsatellites from plants? Mol. Ecol. https://doi.org/10.1046/j.1365-294X.2003.01825.x

Tang, J., Baldwin, S.J., Jacobs, J.M.E., van der Linden, C.G., Voorrips, R.E., Leunissen, J.A.M., van Eck, H., Vosman, B., 2008. Large-scale identification of polymorphic microsatellites using an in silico approach. BMC Bioinformatics. https://doi.org/10.1186/1471-2105-9-374

Thiel, T., Michalek, W., Varshney, R.K., Graner, A., 2003. Exploiting EST databases for the development and characterization of gene-derived SSR-markers in barley (Hordeum vulgare L.). Theor. Appl. Genet. https://doi.org/10.1007/s00122-002-1031-0

Touma, J., García, K.K., Bravo, S., Leiva, F., Moya, J., Vargas-Chacoff, L., Reyes, A., Vidal, R., 2019. De novo Assembly and Characterization of Patagonian Toothfish Transcriptome and Develop of EST-SSR Markers for Population Genetics. Front. Mar. Sci. https://doi.org/10.3389/fmars.2019.00720

Wang, I.J., Summers, K., 2009. Highly polymorphic microsatellite markers for the highly polymorphic strawberry poison-dart frog and some of its congeners. Conserv. Genet. https://doi.org/10.1007/s10592-009-9887-z 
Wang, J., Yu, L., Bai, J., Fan, J., Quan, Y., 2011. Development of EST-SSR markers and analysis of genetic diversity in five populations of grass carp (Ctenopharyngodon idella). Chinese J. Zool. 46(5), 24-32.

Wu, T., Luo, S., Wang, R., Zhong, Y., Xu, X., Lin, Y., He, X., Sun, B., Huang, H., 2014. The first Illumina-based de novo transcriptome sequencing and analysis of pumpkin (Cucurbita moschata Duch.) and SSR marker development. Mol. Breed. https://doi.org/10.1007/s11032-014-0128-X

Xie, Z., Xiao, L., Wang, D., Fang, C., Liu, Q., Li, Z., Liu, X., Zhang, Y., Li, S., Lin, H., 2014. Transcriptome analysis of the Trachinotus ovatus: Identification of reproduction, growth and immune-related genes and microsatellite markers. PLoS One. https://doi.org/10.1371/journal.pone.0109419

Yang, M., Liang, X.F., Tian, C.X., Gul, Y., Dou, Y.Q., Cao, L., Yu, R., 2012. Isolation and characterization of fifteen novel microsatellite loci in golden mandarin fish (Siniperca scherzeri) Steindachne. Conserv. Genet. Resour. https://doi.org/10.1007/s12686-012-9601-1 Yang, Q., Gao, T., Liu, J.X., 2014. Development and characterization of 17 microsatellite loci in an anadromous fish Coilia nasus. Conserv. Genet. Resour. https://doi.org/10.1007/s12686013-0093-4

Zheng, Y., Zhang, G., Lin, F., Wang, Z., Jin, G., Yang, L., Wang, Y., Chen, X., Xu, Z., Zhao, X., Wang, H., Lu, J., Lu, G., Wu, W., 2008. Development of microsatellite markers and construction of genetic map in rice blast pathogen Magnaporthe grisea. Fungal Genet. Biol. https://doi.org/10.1016/j.fgb.2008.07.012 


\section{Figure 1}

Schematic diagram of the principles of SSREnricher.

The program first performs SSR mining and sequence clustering on the sequences, and then combines the two results to enrich the polymorphic SSRs.

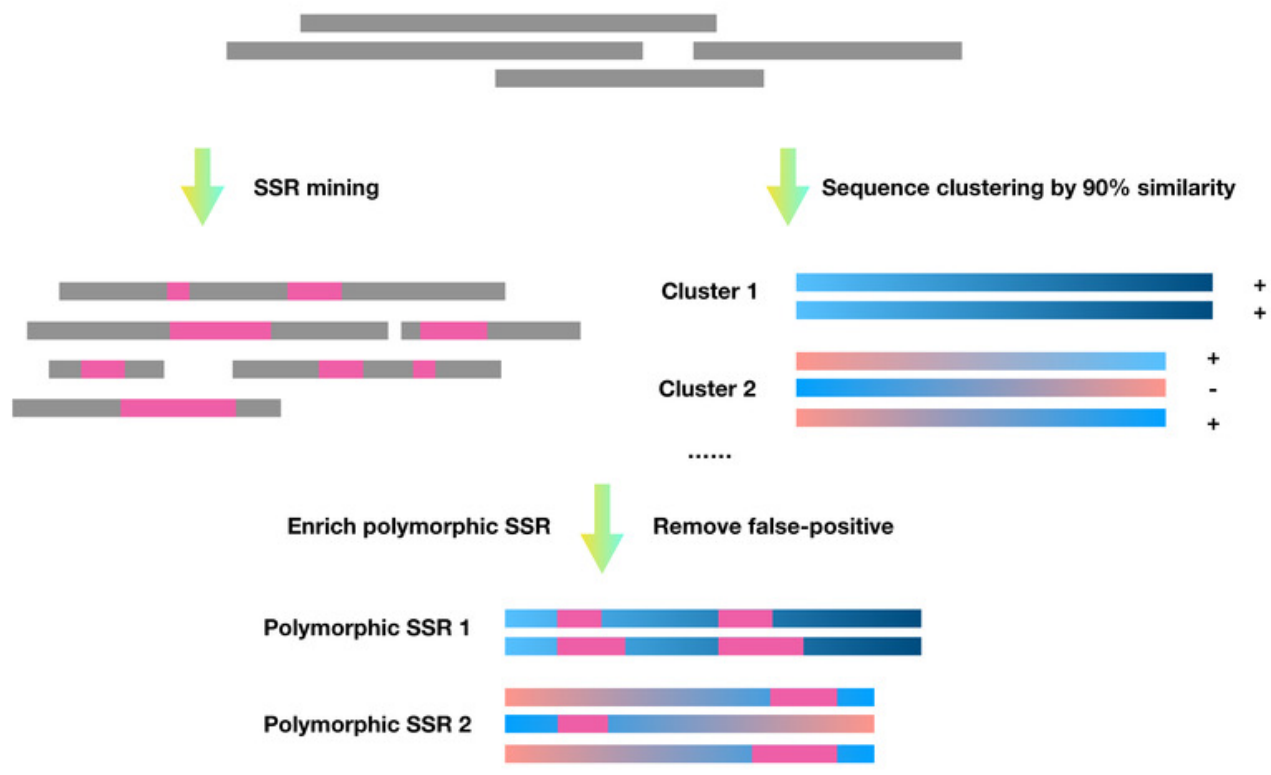


Figure 2

Program flow chart of SSREnricher. 


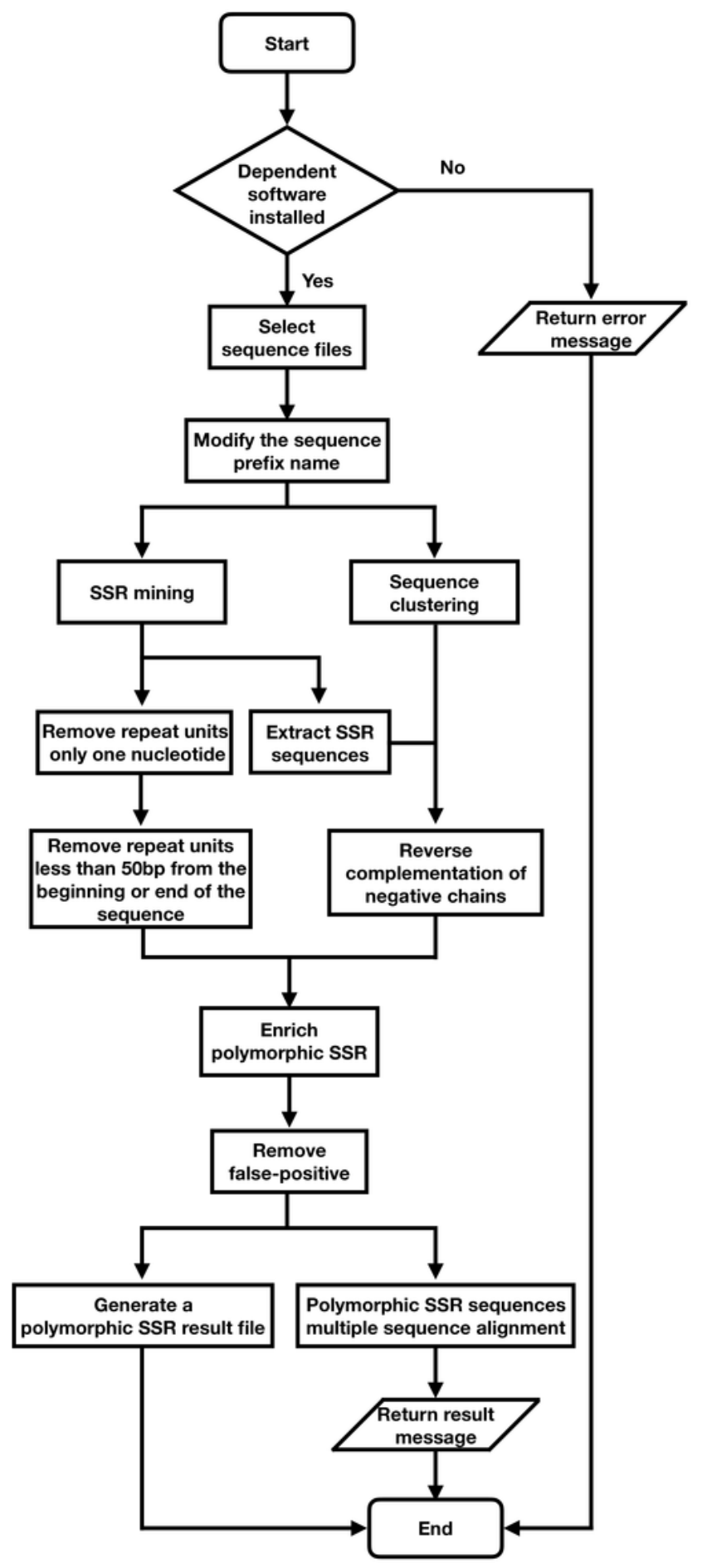




\section{Figure 3}

Comparison of polymorphic marker frequency developed by different methods.

\# represents SSRs developed by HTS; † represents SSRs developed by this method; * and ** represents significance with $P<0.05$ and $P<0.01$ by ANOVA, respectively. The organisms along with their ratio of polymorphic SSR markers used in this figure were as follows: Kuruma prawn (Penaeus japonicas)(Moore et al., 1999), Golden mandarin fish (Siniperca scherzeri)(Yang et al., 2012), Bulatmai barbel (Luciobarbus capito)(Longwu et al., 2012), Estuarine tapertail anchovy (Coilia nasus)(Yang et al., 2014), Pseudogyrincheilus prochilus (Shi et al., 2009), Poison-dart frog (Dendrobates pumilio)(Wang and Summers, 2009), Peganum harmala (Han and An, 2009), Milkfish (Chanos chanos)(Santos et al., 2015), Dwarf bulrush (Typha minima)(Csencsics et al., 2010), Tope shark (Galeorhinus galeus)(Chabot and Nigenda, 2011), Pigeonpea (Cajanus cajan)(Dutta et al., 2011), Water striders (Gerris remigis)(Perry and Rowe, 2011), Barley (Hordeum vulgare)(Thiel et al., 2003), Miiuy croaker (Miichthys miiuy)(Che et al., 2014), Carrot (Daucus carota)(Iorizzo et al., 2011), Pumpkin (Cucurbita moschata)(Wu et al., 2014), Ovate pompano (Trachinotus ovatus)(Xie et al., 2014), Swimming crab (Portunus trituberculatus)(Lv et al., 2014), Blue duck (Hymenolaimus malacorhynchos)(Abdelkrim et al., 2009), Rice (Oryza sativa)(Zheng et al., 2008), Grass carp (Ctenopharyngodon idella)(Wang et al., 2011). 


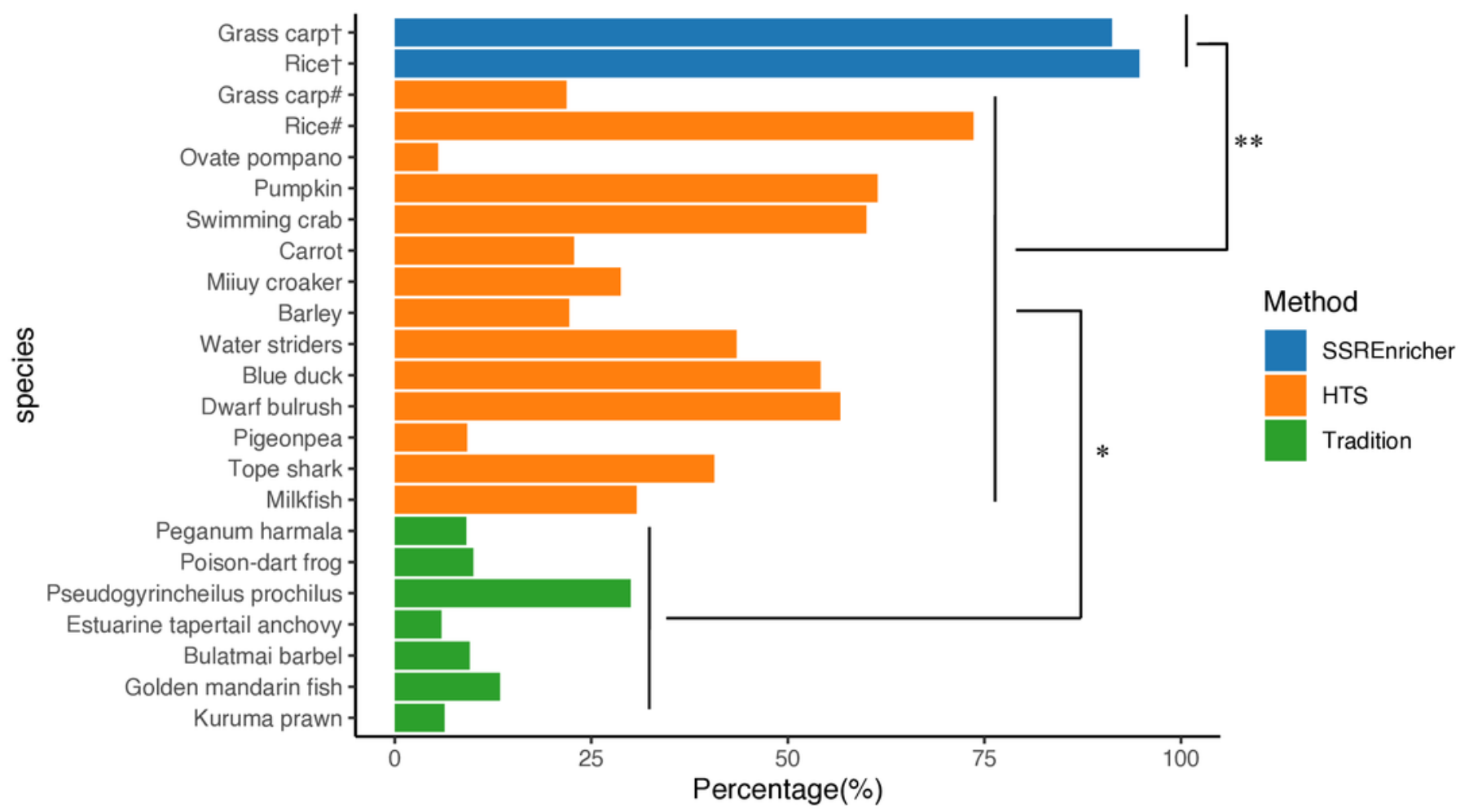




\section{Table $\mathbf{1}$ (on next page)}

Number of SSRs, polymorphic SSRs frequency of two verified species.

Notes: ${ }^{1}$, the mean number of SSRs in the transcriptome (except mononucleotide) SSRs;

${ }^{2}$, the putative polymorphic SSRs enriched by this program were showed in Appendix file 1;

${ }^{3}$, the percentage of primers that can be amplified with clear products bands;

${ }^{4}$, the percentage of SSRs that are polymorphic;

${ }^{5}$, the detail information of polymorphic SSRs validated by experiments was showed in Appendix file 2. 


\begin{tabular}{lll}
\hline & \multicolumn{2}{l}{ Species for verification } \\
\cline { 2 - 3 } & Rice & Grass carp \\
\hline Source of the transcriptome & $\begin{array}{l}\text { SRR1799209 } \\
\text { SRR1974265 } \\
\text { SRR2048540 }\end{array}$ & $\begin{array}{l}\text { SRR1618540 } \\
\text { SRR1618542 }\end{array}$ \\
& 9,901 & 16,700 \\
$\begin{array}{l}\text { Total number of SSRs in transcriptome } \\
\text { (detected by MISA) }\end{array}$ & & $680^{2}$ \\
$\begin{array}{l}\text { Number of putatively polymorphic SSRs } \\
\text { enriched by this program }\end{array}$ & $480^{2}$ & 20 \\
$\begin{array}{l}\text { Number of primers designed for } \\
\text { validation experiments }\end{array}$ & 30 & $20(100 \%)$ \\
$\begin{array}{l}\text { Number of primers amplified with clear } \\
\text { product bands }{ }^{3}(\%)\end{array}$ & $27(90 \%)$ & $19^{5}(95.00 \%)$ \\
\hline \begin{tabular}{l} 
Number of polymorphic SSRs ${ }^{4}(\%)$ \\
\hline
\end{tabular}
\end{tabular}
1 